\title{
In Vitro and In Vivo Evaluation of BO-2727 against Imipenem- and/or Meropenem-resistant Pseudomonas aeruginosa
}

\author{
Kaneyoshi Shibata, Yuka Adachi, Eitchtro Kato, Rie Nagano, Aisaku Fuse, \\ Terutaka Hashizume*, Norikazu Ohtake, Osamu Okamoto \\ and Susumu Nakagawa
}

Tsukuba Research Institute, Banyu Pharmaceutical Co., Ltd., Ohkubo 3, Tsukuba 300-26, Japan

(Received for publication August 5, 1996)

\begin{abstract}
The in vitro and in vivo activity of BO-2727, a carbapenem antibiotic, against resistant clinical isolates of Pseudomonas aeruginosa was studied. The geometric mean MICs against three groups of clinical isolates resistant to imipenem, meropenem and both carbapenems were $4.28,4.08$ and $5.44 \mu \mathrm{g} / \mathrm{ml}$, respectively. BO-2727 also inhibited multiply antibiotic resistant isolates and laboratory mutants including a nalB-type mutant, which showed resistance to antibiotics such as imipenem, meropenem, ceftazidime, and/or ciprofloxacin, at less than $1.56 \mu \mathrm{g} / \mathrm{ml}$. Overall, BO-2727 was 4-fold more active than biapenem, meropenem, panipenem and imipenem with an $\mathrm{MIC}_{90}$ of less than $6.25 \mu \mathrm{g} / \mathrm{ml}$.

The presence of basic amino acids in minimal medium less affected the antipseudomonal activity to a minimal extent, suggesting that BO-2727 has diverse penetration routes through the outer membrane other than OprD channel, which facilitates the diffusion of basic amino acids and carbapenems. The in vitro activity of BO-2727 reflected well in its therapeutic efficacy in experimental systemic infection in mice. These results suggest a possibility for the development of antipseudomonal carbapenems having activity against imipenem- and/or meropenem-resistant $P$. aeruginos $a$ as well as a broad spectrum encompassing Gram-positive and -negative bacteria.
\end{abstract}

Since the discovery of thienamycin ${ }^{1)}$, carbapenem antibiotics are known to have a broad spectrum encompassing both Gram-positive and Gram-negative bacteria including Pseudomonas aeruginosa. However, the recent problems are the emergence of carbapenem-resistant $P$. aeruginosa, in which the mechanisms of resistance are a) deficiency or decreased amount of OprD of the outer membrane, which facilitates the diffusion of carbapenem and basic amino acids ${ }^{2 \sim 5)}$, b) nalB mutation responsible for multiple resistance to cephems, quinolones, tetracyclines as well as meropenem ${ }^{5)}$ and c) the evolution of a class B metallo- $\beta$-lactamase capable of hydrolyzing carbapenems ${ }^{6}$.

BO-2727 is a $1-\beta$-methyl-2-(5-substituted pyrrolidin3-ylthio)carbapenem carrying an (R)-1-hydroxy-3- $N$ methylaminopropyl group as a substituent. We have reported that BO-2727 has a well-balanced, potent, antibacterial spectrum with advantages over meropenem in its antibacterial activity, especially against Grampositive bacteria and $P$. aeruginosa $a^{7,8)}$. BO-2727 showed better activity against $\mathrm{OprD}$ deficient mutants, as previously described ${ }^{9}$.

Our primary concern is directed toward the in vitro and in vivo antipseudomonal activity of BO-2727, especially against imipenem- and/or meropenem-resistant
$P$. aeruginosa. We report that $\mathrm{BO}-2727$ has a high potency against the $P$. aeruginosa isolates resistant to known carbapenems.

\section{Materials and Methods}

Antibiotics

BO-2727, biapenem, meropenem and panipenem were synthesized at the Tsukuba Research Institute, Banyu Pharmaceutical Co., Ltd., Tsukuba, Japan. Imipenem and cilastatin were also the products of Banyu Pharmaceutical Co., Ltd., Tokyo, Japan. Ceftazidime was purchased from Nippon Glaxo Co., Ltd., Tokyo, Japan.

\section{Organisms}

The clinical isolates were from our stock cultures which have been collected from various districts in Japan. Spontaneous mutants with imipenem-resistance were isolated by spreading an overnight culture of imipenemsusceptible parent onto Mueller-Hinton medium agar plate (Difco Laboratories, Detroit, Mich.) containing 3.13 to $25 \mu \mathrm{g} / \mathrm{ml}$ of imipenem. Colonies on the agar plate containing the highest concentration of imipenem were purified on the same concentration of imipenem. The OprD deficiency in the outer membrane proteins of the resistant mutants was confirmed by SDS-polyacrylamide gel electrophoresis ${ }^{2)}$.

Five strains showing meropenem-resistant, but para- 
doxically imipenem-susceptible were screened from our collections of clinical isolates. The nalB mutant was isolated by spreading $P$. aeruginosa PAOl onto an agar plate containing ciprofloxacin and cefsulodin $(1 \mu \mathrm{g} / \mathrm{ml}$ each $)^{5)}$.

\section{MIC Determination}

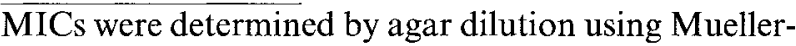
Hinton medium. The culture grown at $37^{\circ} \mathrm{C}$ for 20 hours was diluted to $3 \times 10^{6} \mathrm{CFU} / \mathrm{ml}$, and about $10^{4} \mathrm{CFU} / \mathrm{ml}$ were spotted onto agar plates containing serial twofold dilutions of antibiotics with a replicating device (Microplanter; Sakuma Seisakusho, Tokyo, Japan). The plates were incubated at $37^{\circ} \mathrm{C}$ for 20 hours. The MIC was defined as the lowest concentration of antibiotics which prevented visible growth.

\section{Systemic Infection}

CD-1 male mice, 4 weeks old, were intraperitoneally infected with two imipenem-resistant $P$. aeruginosa, strain BB6265 and BB6268, which were suspended in 5\% gastric mucin. Antibiotics were dissolved in saline, and subcutaneously administered to the mice once at 2 hour after infection. The therapeutic efficacy $\left(E_{50}\right)$ was calculated by probit method from the survival rate on the day 4 after treatment. Cilastatin (CS, DHP-I inhibitor) was co-administered in cases of treatment with meropenem and imipenem due to the instability to the DHP-I ${ }^{10)}$.

\section{Results and Discussion}

\section{Susceptibility of Clinical Isolates of $P$. aeruginosa to Antibiotics}

BO-2727 inhibited all the $P$. aeruginosa tested including imipenem-, meropenem- and/or ceftazidimeresistant in vitro mutants as well as imipenem susceptible isolates at less than $6.25 \mu \mathrm{g} / \mathrm{ml}$ (Table 1). BO-2727 had an $\mathrm{MIC}_{90}$ of $1.56 \mu \mathrm{g} / \mathrm{ml}$ against imipenem-susceptible $P$. aeruginosa. BO-2727 was 4-fold more active in terms of $\mathrm{MIC}_{90}$ than biapenem, meropenem, panipenem and imipenem against imipenem- and/or meropenem-resistant isolates. As shown in the Table 1, the imipenemresistant isolates had reduced susceptibility to all the carbapenems tested, but retrieved appreciable susceptibility to $\mathrm{BO}-2727$ with an $\mathrm{MIC}_{90}$ of $6.25 \mu \mathrm{g} / \mathrm{ml}$. The imipenem-resistant isolates exhibited cross-resistance to

Table 1. Comparative activity against clinical isolates of $P$. aeruginosa.

\begin{tabular}{|c|c|c|c|c|c|}
\hline \multirow{2}{*}{ Isolates (No.) } & \multirow{2}{*}{ Antibiotic } & \multicolumn{4}{|c|}{$\operatorname{MIC}(\mu \mathrm{g} / \mathrm{ml})$} \\
\hline & & Range & G-Mean ${ }^{\mathrm{a}}$ & $50 \%$ & $90 \%$ \\
\hline \multirow[t]{6}{*}{ Imipenem-susceptible (128) } & BO- 2727 & $0.1 \sim 6.25$ & 0.54 & 0.39 & 1.56 \\
\hline & Biapenem & $0.2 \sim 3.13$ & 0.80 & 0.78 & 1.56 \\
\hline & Meropenem & $0.1 \sim 25$ & 0.54 & 0.39 & 3.13 \\
\hline & Panipenem & $0.39 \sim 25$ & 5.86 & 6.25 & 12.5 \\
\hline & Imipenem & $0.2 \sim 6.25$ & 1.63 & 1.56 & 3.13 \\
\hline & Ceftazidime & $0.39 \sim>100$ & 3.74 & 3.13 & 25 \\
\hline \multirow{6}{*}{ Imipenem-resistant (22) } & BO- 2727 & $1.56 \sim 6.25$ & 4.28 & 3.13 & 6.25 \\
\hline & Biapenem & $6.25 \sim 25$ & 14.6 & 12.5 & 25 \\
\hline & Meropenem & $1.56 \sim 50$ & 7.09 & 6.25 & 25 \\
\hline & Panipenem & $6.25 \sim 50$ & 24.5 & 25 & 50 \\
\hline & Imipenem & $12.5 \sim 50$ & 16.6 & 12.5 & 25 \\
\hline & Ceftazidime & $0.39 \sim 50$ & 9.72 & 12.5 & 50 \\
\hline \multirow{6}{*}{ Meropenem-resistant (13) } & BO-2727 & $0.78 \sim 6.25$ & 4.08 & 6.25 & 6.25 \\
\hline & Biapenem & $0.78 \sim 25$ & 11.2 & 12.5 & 25 \\
\hline & Meropenem & $12.5 \sim 50$ & 17.2 & 12.5 & 25 \\
\hline & Panipenem & $12.5 \sim 50$ & 23.7 & 25 & 25 \\
\hline & Imipenem & $1.56 \sim 25$ & 11.2 & 12.5 & 25 \\
\hline & Ceftazidime & $3.13 \sim>100$ & 23.7 & 25 & 50 \\
\hline \multirow{6}{*}{$\begin{array}{l}\text { Imipenem- and meropenem- } \\
\text { resistant (10) }\end{array}$} & BO- 2727 & $3.13 \sim 6.25$ & 5.44 & 6.25 & 6.25 \\
\hline & Biapenem & $12.5 \sim 25$ & 18.9 & 25 & 25 \\
\hline & Meropenem & $12.5 \sim 50$ & 17.7 & 12.5 & 25 \\
\hline & Panipenem & $25 \sim 50$ & 26.8 & 25 & 25 \\
\hline & Imipenem & $12.5 \sim 25$ & 16.5 & 12.5 & 25 \\
\hline & Ceftazidime & $3.13 \sim 50$ & 20.3 & 25 & 50 \\
\hline \multirow[t]{6}{*}{ Ceftazidime-resistant (39) } & BO-2727 & $0.2 \sim 6.25$ & 1.43 & 1.56 & 6.25 \\
\hline & Biapenem & $0.39 \sim 25$ & 2.81 & 1.56 & 25 \\
\hline & Meropenem & $0.2 \sim 50$ & 2.48 & 3.13 & 25 \\
\hline & Panipenem & $1.56 \sim 50$ & 11.4 & 12.5 & 25 \\
\hline & Imipenem & $0.78 \sim 50$ & 4.01 & 3.13 & 25 \\
\hline & Ceftazidime & $12.5 \sim>100$ & 31.5 & 25 & 100 \\
\hline
\end{tabular}

a Geometric mean. 
biapenem and panipenem. Interestingly, meropenem was active against some of the imipenem-resistant isolates, as shown by the susceptibility range of 1.56 to $50 \mu \mathrm{g} / \mathrm{ml}$, although certain meropenem-resistant isolates showing cross-resistance to imipenem. Thus, $P$. aeruginosa isolates showing carbapenem-resistance were roughly grouped in the imipenem- and/or meropenem-resistance (Table 1). Susceptibility testing against the 13 isolates resistant to meropenem, and 10 isolates resistant to imipenem and meropenem (both $\mathrm{MIC} ; \geq 12.5 \mu \mathrm{g} / \mathrm{ml}$ ) also displayed the high potency of $\mathrm{BO}-2727$ with geometric mean MIC of 4.08 and $5.44 \mu \mathrm{g} / \mathrm{ml}$, respectively. Furthermore, BO-2727 was more active than ceftazidime against ceftazidimeresistant isolates, which included imipenem- and/or meropenem-resistant isolates. As a result, BO-2727 inhibited all the $P$. aeruginosa resistant to imipenem, meropenem and/or ceftazidime at less than $6.25 \mu \mathrm{g} / \mathrm{ml}$.

\section{Susceptibility of Multiply Resistant \\ $P$. aeruginosa to Antibiotics}

Table 2 shows the susceptibility of meropenemresistant, but paradoxically imipenem-susceptible, clini- cal isolates and of a nalB mutant derived from strain PAO1 which were associated with multiple resistance to ceftazidime and ciprofloxacin, and called nalB-type isolates ${ }^{5)}$. The five nalB-type isolates and the laboratory mutant were more susceptible to $\mathrm{BO}-2727$ than to the other carbapenems tested. The nalB mutation affected the susceptibility to meropenem, but not to other carbapenems.

\section{Effect of Basic Amino Acids in Medium on the Antipseudomonal Activity}

The decreased susceptibility of carbapenems in minimal medium with rich basic amino acids relates to increased competition for diffusion of carbapenems through OprD, as previously reported ${ }^{3,4)}$. The geometric mean MICs of $\mathrm{BO}-2727$, meropenem, panipenem, and imipenem against the imipenem-susceptible strains were 2.7-, 7.2-, 5.3-, and 3.5-fold increased by the presence of $50 \mathrm{~mm}$ L-lysine in the minimal medium, respectively. The activity of BO-2727 was less affected by basic amino acids than a positive agent like meropenem, suggesting a lesser degree of dependency on OprD for penetration through

Table 2. Comparative activity against meropenem-resistant $P$. aeruginosa including a nalB mutant.

\begin{tabular}{|c|c|c|c|c|c|c|c|}
\hline \multirow{2}{*}{ Strain } & \multicolumn{7}{|c|}{$\operatorname{MIC}(\mu \mathrm{g} / \mathrm{ml})$} \\
\hline & BO- 2727 & Biapenem & Meropenem & Panipenem & Imipenem & Ceftazidime & Ciprofloxacin \\
\hline \multicolumn{8}{|l|}{ (A) Clinical isolates } \\
\hline BB6176 & 0.78 & 0.78 & 12.5 & 12.5 & 1.56 & 12.5 & 6.25 \\
\hline BB6276 & 0.78 & 3.13 & 25 & 12.5 & 3.13 & $>100$ & 3.13 \\
\hline BB6277 & 0.39 & 0.78 & 6.25 & 1.56 & 0.78 & 6.25 & 100 \\
\hline BB6285 & 1.56 & 3.13 & 12.5 & 25 & 6.25 & 25 & 0.78 \\
\hline BB6287 & 0.78 & 1.56 & 6.25 & 3.13 & 3.13 & $>100$ & 3.13 \\
\hline \multicolumn{8}{|c|}{ (B) nalB mutant derived from strain PAO1 } \\
\hline PAO1 (parent) & 1.56 & 1.56 & 0.78 & 6.25 & 3.13 & 0.39 & 0,05 \\
\hline BB6292 (mutant) $)^{\mathrm{a}}$ & 1.56 & 1.56 & 3.13 & 6.25 & 3.13 & 3.13 & 0,2 \\
\hline
\end{tabular}

a The mutant was isolated by spreading $P$. aeruginosa PAOI onto an agar plate containing ciprofloxacin and cefsulodin $(1 \mu \mathrm{g} / \mathrm{ml}$ each) $)^{5}$.

Table 3. Effect of a basic amino acid on the antipseudomonal activity of BO-2727 and reference $\beta$-lactam antibiotics.

\begin{tabular}{|c|c|c|c|c|c|c|}
\hline \multirow{2}{*}{$\begin{array}{l}\text { Strain (No.) } \\
\text { Medium }\end{array}$} & \multicolumn{6}{|c|}{ Geometric mean $\mathrm{MIC}(\mu \mathrm{g} / \mathrm{ml})$} \\
\hline & $\mathrm{BO}-2727$ & Biapenem & Meropenem & Panipenem & Imipenem & Ceftazidime \\
\hline \multicolumn{7}{|l|}{ Imipenem-susceptible (12) } \\
\hline Minimal medium ${ }^{\mathrm{a}}$ & 0.22 & 0.25 & 0.082 & 0.74 & 0.44 & 1.97 \\
\hline Minimal medium with lysine & $0.59(2.7)^{\mathrm{b}}$ & $0.78(3.1)$ & $0.59(7.2)$ & $3.94(5.3)$ & $1.56(3.5)$ & $1.97(1.0)$ \\
\hline Mueller-Hinton medium & 0.52 & 0.66 & 0.93 & 4.96 & 1.47 & 7.43 \\
\hline \multicolumn{7}{|c|}{ Imipenem-resistant isogenic mutants $(12)^{\mathrm{c}}$} \\
\hline Mueller-Hinton medium & 3.72 & 13.2 & 9.36 & 22.3 & 16.7 & 7.43 \\
\hline
\end{tabular}

a Modified Davis minimal medium supplemented with $0.4 \%$ sodium gluconate and solidified with $1.5 \%$ agar ${ }^{4}$.

b Ratio of MIC in minimal medium with $50 \mathrm{~mm}$ L-lysine to that in minimal medium alone for indicated antibiotics.

c In vitro imipenem-resistant mutants spontaneously isolated from imipenem-susceptible strains. 
Table 4. Therapeutic efficacy against systemic infections in mice.

\begin{tabular}{clcc}
\hline $\begin{array}{c}\text { Strain } \\
(\text { CFU/mouse })\end{array}$ & Antibiotic $^{\mathrm{a}}$ & $\begin{array}{c}\text { MIC } \\
(\mu \mathrm{g} / \mathrm{ml})\end{array}$ & $\begin{array}{c}\text { ED }_{50}(95 \% \\
\text { confidence limits })^{\mathrm{b}} \\
(\mathrm{mg} / \mathrm{kg})\end{array}$ \\
\hline $\begin{array}{c}\text { P. aeruginosa } \text { BB6265 } \\
2.9 \times 10^{6}\left(4.4 \times \mathrm{LD}_{50}\right)\end{array}$ & BO-2727 & 1.56 & $2.09(0.79 \sim 5.73)$ \\
& Biapenem & 6.25 & $3.00(1.49 \sim 5.71)$ \\
& Meropenem/CS & 3.13 & $6.06(2.41 \sim 15.6)$ \\
& Imipenem/CS & 12.5 & $7.62(1.15 \sim 19.3)$ \\
Ceftazidime & 0.78 & $7.15(2.38 \sim 16.8)$ \\
$9.5 \times 10^{5}\left(37 \times\right.$ LD $\left._{50}\right)$ & BO-2727 & 1.56 & $3.33(1.28 \sim 7.29)$ \\
& Biapenem & 3.13 & $8.05(3.94 \sim 17.8)$ \\
& Meropenem $/ \mathrm{CS}$ & 6.25 & $17.4(7.99 \sim 40.3)$ \\
& Imipenem/CS & 12.5 & $12.0(6.33 \sim 21.3)$ \\
& Ceftazidime & 3.13 & $26.6(9.89 \sim 79.1)$ \\
\hline
\end{tabular}

a Administered subcutaneously at 2 hours after i.p. infection.

b Calculated by the probit method.

c Cilastatin (CS) was coadministered ${ }^{10}$.

the outer membrane in $P$. aeruginosa.

\section{In Vivo Efficacy against Experimental Pseudomonal Infections}

BO-2727 exhibited good efficacy in systemic infection with imipenem-resistant $P$. aeruginosa, which reflected the in vitro activity (Table 4 ). Ceftazidime showed weaker in vivo activity than could be expected from its in vitro activity (the MIC; $3.13 \mu \mathrm{g} / \mathrm{ml}$ ) in spite of lack of crossresistance between imipenem and ceftazidime based on their different mechanisms of resistance. The results suggested a possibility for the antipseudomonal agent having the activity against imipenem- and/or meropenem-resistant $P$. aeruginosa as well as broad spectrum encompassing Gram-positive and Gram-negative bacteria $^{11,12)}$.

\section{References}

1) Kahan, J. S.; F. M. Kahan, R. Goegelman, S. A. Currie, M. Jackson, E. O. Stapley, T. W. Miller, A. K. Miller, D. Hendlin, S. Mochales, S. Hernandez, H. B. WOODRUFF \& J. BIRNBAUM: Thienamycin, a new $\beta$-lactam antibiotic. I. Discovery, taxonomy, isolation and physical properties. J. Antibiotics 32: 1 12, 1979

2) Quinn, J. P.; A. Darzins, D. MiYashiro, S. Ripp \& R. W. Miller: Imipenem resistance in Pseudomonas aeruginosa PAO: Mapping of the OprD2 gene. Antimicrob. Agents Chemother. 35: 753 755, 1991

3) Trias, J. \& H. NiKaido: Outer membrane protein D2 catalyzes facilitated diffusion of carbapenems and penems through the outer membrane of Pseudomonas aeruginosa. Antimicrob. Agents Chemother. 34: 52 57, 1990

4) Fukuoka, T.; N. Masuda, T. Takeñouchi, N. Sekine, M. IJIma \& S. OHYA: Increase in susceptibility of Pseudomonas aeruginosa to carbapenem antibiotics in low-amino-acid media. Antimicrob. Agents Chemother. 35: $529 \sim 532,1991$
5) Masuda, N. \& S. Ohaya: Cross resistance to meropenem, cephems and quinolones in Pseudomonas aeruginosa. Antimicrob. Agents Chemother. 36: 1847 1851, 1992

6) Saino, Y.; F. Kobayashi, M. Inoue \& S. Mitsuhashi; Purification and properties of inducible penicillin $\beta$-lactamase isolated from Pseudomonas maltophilia. Antimicrob. Agents Chemother. 22: $564 \sim 570,1982$

7) Nakagawa, S.; T. Hashizume, K. Matsuda, M. Sanada, O. OKamoto, H. FuKatsu \& N. TanaKa: In vitro activity of a new carbapenem antibiotic, BO-2727, with potent anti-pseudomonal activity. Antimicrob. Agents Chemother. 37: $2756 \sim 2759,1993$

8) Asahi, Y.; S. Miyazaki \& K. Yamaguchi: In vitro and in vivo antibacterial activities of $\mathrm{BO}-2727$, a new carbapenem. Antimicrob. Agents Chemother. 39: 1030 1037,1995

9) Hazumi, N.; A. Fuse, K. Matsuda, T. Hashizume \& M. SANADA: Mechanism of enhanced antipseudomonal activity of $\mathrm{BO}-2727$, a new injectable 1- $\beta$-methyl carbapenem. Antimicrob. Agents Chemother. 39: $702 \sim$ 706,1995

10) Fukasawa, M.; Y. Sumita, E. T. Harabe, T. Tanio, H. Nouda, T. Kohzuki, T. OKuda, H. Matsumura \& M. SunaGawa: Stability of meropenem and effect of $1 \beta$-mehyl substitution on its stability in the presence of renal dehydropeptidase I. Antimicrob. Agents Chemother. 36: $1577 \sim 1579,1992$

11) Hashizume, T.; N. Hazumi, K. Shibata, H. Miyazaki, Y. Adachi, M. Sanada, S. Nakagawa \& N. Tanaka: Antipseudomonal activity of BO-2727 against imipenemand/or meropenem-resistant Pseudomonas aeruginosa. The 34th Interscience Conference on Antimicrobial Agents and Chemotherapy, abstract F-11, October 4 7, Orlando, Florida, 1994

12) Kohler, J.; B. A. Pelak, L. S. Gerckens, J. S. Kahan \& H. KRoPP: Comparative in vitro resistance studies with carbapenems BO-2727 against Pseudomonas aeruginosa. 1993. The 33rd Interscience Conference on Antimicrobial Agents and Chemotherapy, abstract $\$ 902$, October $17 \sim 20$, New Orleans, Louisiana, 1993 